\title{
Effects of Imperfect Subcarrier SNR Information on Adaptive Bit Loading Algorithms for Multicarrier Systems
}

\author{
Alexander M. Wyglinski \\ Fabrice Labeau \\ Peter Kabal \\ Electrical \& Computer Engineering, McGill University \\ 3480 University St., Montréal, Québec, Canada H3A 2A7 \\ Email: alexw@TSP.ECE.McGill.CA
}

\begin{abstract}
In this paper, we evaluate and compare the robustness of several adaptive bit loading algorithms for multicarrier transmission systems when imperfect subcarrier signal-to-noise ratio (SNR) information is used. In particular, we investigate the impact of the uncertainty of data-aided channel estimation techniques on system performance. We also examine an implementation issue associated with adaptive bit loading algorithms that use metrics related to the SNR. Although such metrics can be derived via closed form expressions, look-up tables are used instead to reduce system complexity, resulting in the SNR values being quantized. Thus, we examine the effects of SNR quantization on system performance. Finally, we present a technique for choosing SNR values in a fixed length look-up table in order to minimize quantization error.
\end{abstract}

Keywords:

Adaptive modulation, multicarrier communications, imperfect channel estimation

\section{INTRODUCTION}

Multicarrier modulation (MCM) is increasingly being employed in many high-speed data transmission systems, including several wireless local area network (WLAN) standards [1]. The advantage of MCM is that it can transmit data at lower rates on each subcarrier simultaneously. As a result, the frequency-selective fading channel is effectively transformed into a collection of nearly flat-fading subchannels. Many of these MCM systems, especially the wireless ones [1], use conventional multicarrier modulation which employs the same signal constellation across all subcarriers. These suffer from the subcarriers with the poorest error performance. One solution is to perform adaptive "bit loading", where the signal constellation size across the subcarriers varies. In extreme situations, some subcarriers can be "turned off" or nulled if the subcarrier SNR values are poor.

There have been numerous studies on the performance of multicarrier systems that employ adaptive bit loading algorithms, where the subcarrier SNR information is assumed to be perfectly known [2-6]. However, these results are overly optimistic since they neglected the degree of uncertainty that exists with the subcarrier SNR information. In an attempt to provide more accurate results, several studies have included models of these sources of uncertainty, such as noisy channel estimates $[7,8]$ and outdated channel estimates due to time varying channels $[8,9]$.

This research was partially funded by the Natural Sciences and Engineering Research Council of Canada (NSERC) and Le Fonds de Recherche sur la Nature et les Technologies du Québec.

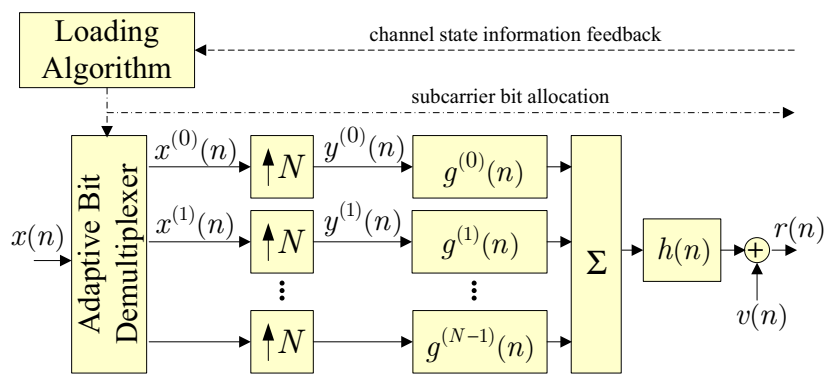

(a) Transmitter with adaptive bit loading algorithm and channel

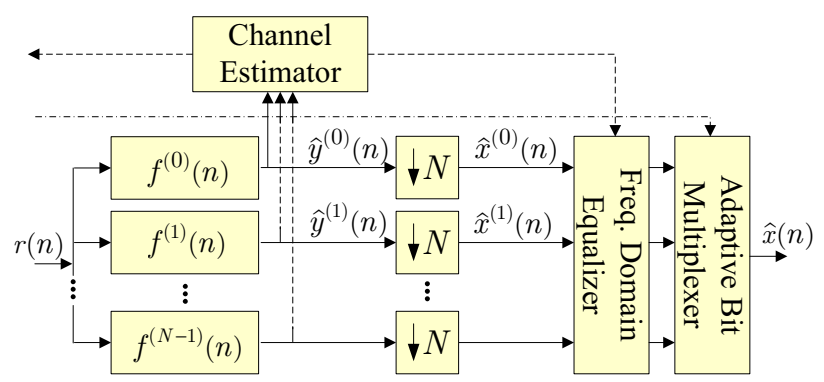

(b) Receiver with channel estimator

Fig. 1 Schematic of an adaptive multicarrier modulation system in the downlink direction using feedback from the channel estimator.

In this paper, we present a comparative study of the robustness of four adaptive bit loading algorithms with imperfect subcarrier SNR information. The adaptive MCM system used in this work is presented in Section II while the channel estimation technique and channel estimation error model are presented in Sections III and IV. Section VI briefly describes the adaptive bit loading algorithms studied in this paper.

Since adaptive bit loading algorithms use metrics that are functions of the subcarrier SNR, such the bit error rate (BER) [4-6], a look-up table of metric values can be used to reduce complexity. However, the size of the look-up table as well as the metric values chosen to be stored can impact system performance by introducing quantization error into the subcarrier SNR information. In this work, the effects of subcarrier SNR quantization due to a finite-size look-up table is studied. Section IV presents the SNR quantization noise model employed in this paper. Furthermore, a technique is presented in Section $V$ that chooses the subcarrier SNR/BER pairs for the look-up table given a number of modulation schemes in order to minimize the quantization error. The simulation results 
are presented and discussed in Section VII, while concluding remarks are made in Section VIII.

\section{Adaptive Multicarrier Modulation}

The general setup for an adaptive MCM system is shown in Fig. 1. The high speed input symbol stream, $x(n)$, is demultiplexed into $N$ streams, with stream $i$ having $b_{i}$ bits per symbol epoch. The value of $b_{i}$ is determined by the adaptive bit loading algorithm, which uses the subcarrier SNR values $\gamma_{i}, i=0, \ldots, N-1$, to compute the subcarrier BER $[5,6]$ or the channel capacity [3]. The subcarrier SNR values are computed from the channel state information (CSI) provided by the data-aided channel estimator at the receiver. We consider the downlink in this paper, with adaptive bit loading performed solely at the transmitter.

Once the bit streams are modulated onto one of several signal constellations consisting of $M_{i}=2^{b_{i}}$ points, the outputs, $x^{(i)}(n), i=0, \ldots, N-1$, are upsampled by a factor $N$ to produce $y^{(i)}(n), i=0, \ldots, N-1$, and filtered by synthesis filters $g^{(i)}(n), i=0, \ldots, N-1$, before being summed together, yielding the composite transmit signal, $s(n)$. This signal is transmitted across the channel, where the multipath propagation and additive noise are modelled with channel impulse response $h(n)$ and noise $v(n)$. The received signal, $r(n)$, is separated into the $N$ subchannels using the analysis filters $f^{(i)}(n)$, $i=0, \ldots, N-1$, downsampled by a factor $N$, equalized using frequency-domain equalizers, demodulated, and then multiplexed together to form the estimate of $x(n), \hat{x}(n)$.

\section{DATA-Aided CHANNEL ESTIMATION}

The deviation between the actual bit allocation and the optimal allocation is dependent on the adaptive bit loading algorithm employed and the quality of the CSI. This quality is partially dependent on the channel estimation technique. In this work, data-aided channel estimation is employed, where the transmission of data is interrupted as needed in order for training symbols to be sent across the channel. At the receiver, the training symbols are extracted and a channel estimate is communicated back to the transmitter using the reverse channel. Thus, the data throughput decreases due to increase in transmission overhead.

To perform data-aided channel estimation in a multicarrier system, we refer to Fig. 1 . Let $x^{(i)}(n), i=0, \ldots, N-1$, be a collection of BPSK-modulated training signals which are known at the receiver. Using the $K$-point discrete Fourier transform (DFT),

$$
X(m) \triangleq \sum_{n=0}^{K-1} x(n) e^{-j 2 \pi n m / K}, \quad 0 \leq m \leq K-1
$$

where $K$ is sufficiently long. We apply Eq. (1) to $y^{(i)}(n)$, $g^{(i)}(n), h(n), v(n)$, and $f^{(i)}(n)$, which yields $Y^{(i)}(m)$, $G^{(i)}(m), H(m), V(m)$, and $F^{(i)}(m)$, respectively.

When no noise is present and the channel impulse response consists of a delta, the output of the $i^{\text {th }}$ analysis filter in terms of $Y^{(i)}(m)$ is given by

$$
\hat{Y}_{\text {Ideal }}^{(i)}(m)=F^{(i)}(m) \sum_{k=0}^{N-1} G^{(k)}(m) Y^{(k)}(m) .
$$

However, when a dispersive channel $h(n)$ and noise $v(n)$ are present in the system, Eq. (2) becomes

$$
\begin{aligned}
\hat{Y}^{(i)}(m)= & F^{(i)}(m) H(m) \sum_{k=0}^{N-1} G^{(k)}(m) Y^{(k)}(m) \\
& +F^{(i)}(m) V(m) \\
= & H(m) \hat{Y}_{\text {Ideal }}^{(i)}(m)+F^{(i)}(m) V(m) .
\end{aligned}
$$

Given that $y^{(i)}(n), g^{(i)}(n)$, and $f^{(i)}(n)$ are known at the receiver, the channel estimate in the passband of subcarrier $i$, with the frequency range corresponding to the bins $m_{L}^{(i)} \leq$ $m<m_{U}^{(i)}$, is computed using [10]:

$$
\begin{aligned}
\hat{H}^{(i)}(m) & =\frac{\hat{Y}^{(i)}(m)}{\hat{Y}_{\text {Ideal }}^{(i)}(m)}=\frac{\hat{Y}^{(i)}(m) \hat{Y}_{\text {Ideal }}^{(i) *}(m)}{\left|\hat{Y}_{\text {Ideal }}^{(i)}(m)\right|^{2}} \\
& =H(m)+\frac{F^{(i)}(m) V(m) \hat{Y}_{\text {Ideal }}^{(i) *}(m)}{\left|\hat{Y}_{\text {Ideal }}^{(i)}(m)\right|^{2}} \\
& =H(m)+\Delta^{(i)}(m),
\end{aligned}
$$

where $\hat{H}^{(i)}(m)$ is the $K$-point DFT of the channel estimate $\hat{h}^{(i)}(n), \Delta^{(i)}(m)$ is the channel estimation error associated with subcarrier $i$, and $m_{L}^{(i)}$ (resp. $m_{U}^{(i)}$ ) is the frequency bin corresponding to the lowest (resp. highest) frequency portion of the passband for subcarrier $i$. The estimate of the entire channel response is simply the sum of Eq. (4) across all the subcarriers, which yields

$$
\hat{H}(m)=H(m)+\sum_{i=0}^{N-1} \Delta^{(i)}(m) .
$$

From Eq. (5), it is observed that the $\Delta^{(i)}(m)$ term represents the uncertainty of the channel estimation procedure. Therefore, the accuracy of the channel estimation is dependent on the amount of noise present in the channel as well as on the choice of synthesis and analysis filters used by the system.

The system performance is also affected by the time variation of the channel and the rate at which the channel estimate is updated $[8,9]$. When the channel varies rapidly over time, the rate at which the channel estimate needs to be updated must also be high, resulting in increased transmission overhead. If the update rate is too low, the system will use outdated channel estimates which may lead to significant performance degradation. On the other hand, if the rate is too high, the data throughput significantly decreases (with no additional performance gain). Thus, the dynamics of the channel must be known in order to determine the appropriate channel estimate update rate. In this work, we will use examples of indoor wireless networks, where the channel is assumed to be quasi-stationary due to the low velocities of the mobiles in this environment [10]. Therefore, we can assume that the channel is time invariant over a reasonably long period of time.

\section{MODELS FOR IMPERFECT SNR INFORMATION}

\section{A. Gaussian Subcarrier SNR Noise Model}

To model the effect of channel estimation errors in the subcarrier SNR information, we refer to Eq. (4). It is observed 
that the channel estimation error consists of the term $\Delta^{(i)}(m)$. Therefore, using Eq. (4), the SNR for subcarrier $i$ based on channel estimates is given by

$$
\begin{aligned}
\hat{\gamma}^{(i)}= & \frac{1}{M_{\text {bins }}^{(i)}} \sum_{m=m_{L}^{(i)}}^{m_{U}^{(i)}} \frac{\pi^{(i)}\left|\hat{H}^{(i)}(m)\right|^{2}}{\sigma_{v}^{2}} \\
= & \frac{1}{M_{\text {bins }}^{(i)}} \sum_{m=m_{L}^{(i)}}^{m_{U}^{(i)}} \frac{\pi^{(i)}\left|H(m)+\Delta^{(i)}(m)\right|^{2}}{\sigma_{v}^{2}} \\
= & \frac{1}{M_{\text {bins }}^{(i)}} \sum_{m=m_{L}^{(i)}}^{m_{U}^{(i)}}\left(\frac{\pi^{(i)}|H(m)|^{2}}{\sigma_{v}^{2}}\right. \\
& \left.+\frac{\pi^{(i)}\left(2 \operatorname{Re}\left\{H(m) \Delta^{(i) *}(m)\right\}+\left|\Delta^{(i)}(m)\right|^{2}\right)}{\sigma_{v}^{2}}\right) \\
= & \gamma^{(i)}+\epsilon^{(i)}
\end{aligned}
$$

where $M_{\text {bins }}^{(i)}=m_{U}^{(i)}-m_{L}^{(i)}+1$ is the number of frequency bins corresponding to the passband of subcarrier $i, \pi^{(i)}$ is the transmit power for subcarrier $i, \sigma_{v}^{2}$ is the noise variance, $\gamma^{(i)}$ is the actual SNR of subcarrier $i$, and $\epsilon^{(i)}$ is the contribution of the channel estimation error to the subcarrier SNR.

The subcarrier channel estimation error $\epsilon^{(i)}$ is approximated by a Normal distribution with zero mean and variance $\sigma^{2}$ [7]. However, we constrained the negative values of $\epsilon^{(i)}$ such that Eq. (6), being the ratio of powers, is never negative.

\section{B. SNR Quantization Noise Model}

Adaptive bit loading algorithms normally use a metric in order to determine the bit allocation. The most commonly used metric is the subcarrier BER, which can be related to the subcarrier SNR via closed form expressions, given that the modulation scheme employed is known. To reduce the implementation complexity, a look-up table can be employed instead to translate subcarrier SNR values into subcarrier BER values, $P_{i}$, for each subcarrier $i$. However, this implies that the subcarrier SNR values must first be quantized before the look-up table is used. This quantization procedure results in additional error that can cause the bit allocation to deviate further from the ideal.

In this work, a mid-rise uniform quantizer is employed to quantize the subcarrier SNR values (in decibels). However, in order to ensure that we minimize the overall error when considering all the modulation schemes, we must determine where to place the quantizer reproduction (i.e. output) levels, $d_{k}$. Since we want adequate resolution of the bit error rate waterfall curves around the target probability of bit error, $P_{T}$, we should concentrate our output levels about that point. In the next section, we present a novel sub-optimal $d_{k}$ placement technique which tries to minimize the overall error while providing adequate resolution.

\section{Quantization Reproduction LeVel Placement TECHNIQUE}

To obtain adequate resolution of the BER waterfall curves for the modulation schemes employed by the system, where

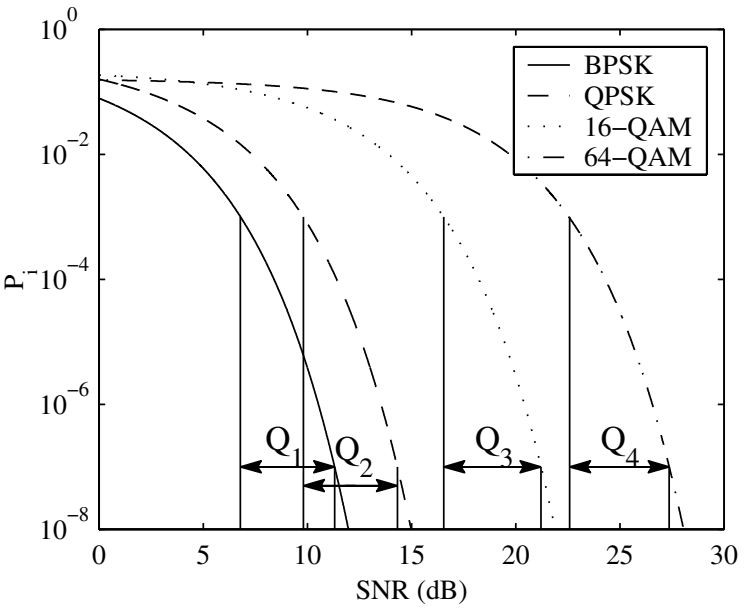

Fig. 2 Regions of uniform quantization of the $P_{i}$ waterfall curves for $P_{T}=10^{-5}$ and $B=4$.

the rate of decrease for each curve many vary drastically, we present the following algorithm that tries to perform a suboptimal placement of $d_{k}$ :

1. Given $q$ bits to represent a quantizer reproduction level, the number of levels is defined as $2^{q}$, which corresponds to a $2^{q}$-entry look-up table.

2. Determine the pair of SNR values to obtain the probability of bit error values, $P_{i}$, that are two orders of magnitude above and below $P_{T}$ for each modulation scheme, thus forming regions $Q_{k}$, for $k=1, \ldots, B$, where $B$ is the number of modulation schemes.

3. For the $B$ modulation schemes, put $2^{q} / B$ output levels uniformly in $Q_{k}$ for all $k$. In the case of overlapping regions, combine them and their allocation of output levels, distributing the levels uniformly across the combined region.

An example of this procedure is shown in Fig. 2 for $P_{T}=10^{-5}$ and $B=4$. In this case, the $P_{i}$ curves correspond to BPSK, QPSK, 16-QAM, and 64-QAM. If $P_{i}>10^{-3}$, quantizing that part of the BER curve is not worthwhile since the $P_{i}$ is so high that the subcarrier would be nulled. On the other hand, if $P_{i}<10^{-7}$, then $P_{i}$ is so far below $P_{T}$ that any quantization would not significantly affect the mean BER of the system, $\bar{P}$. Where $Q_{1}$ and $Q_{2}$ overlap, the output levels allocated to the two regions would be combined and distributed uniformly across the aggregate region.

By distributing $d_{k}, k=0, \ldots, 2^{q}-1$, in this way, we ensure that the BER waterfall curves are quantized with sufficient resolution.

\section{AdAptive Bit LoAding Algorithms}

In this paper, we study the performance of multicarrier systems that employ the adaptive bit loading algorithms of Fox [2], Leke \& Cioffi [3], and the two algorithms of the authors [5, 6]. Fox developed an incremental allocation algorithm and specified conditions for an optimal allocation [2]. The algorithm by Leke \& Cioffi uses an approximation of the channel capacity to determine the bit allocation [3]. Finally, both algorithms by the authors try to attain a target average bit error 
rate. One uses a decremental allocation approach [5], while the other uses an iterative allocation technique [6]. For details and a comparative study of these four algorithms when the subcarrier SNR information is perfectly known, the reader is referred to $[5,6]$.

\section{Simulation Results}

\section{A. Simulation Parameters}

Using the indoor channel model of Saleh \& Valenzuela [11], we employed the adaptive bit loading algorithms in an indoor wireless multicarrier system simulator based on IEEE Std. 802.11a [1]. The transmitter/receiver separation was varied between $1 \mathrm{~m}$ and $60 \mathrm{~m}$, which is reflected by a corresponding change in the overall SNR ranging from $58 \mathrm{~dB}$ to $-9 \mathrm{~dB}$. Furthermore, there was no line-of-sight and the channel was time-invariant. The signal, which is composed of 52 subcarriers, is transmitted across a $16.6 \mathrm{MHz}$ bandwidth. Results are averaged over 10,000 different channel realizations. The system can employ BPSK, QPSK, rectangular 16-QAM, and rectangular 64-QAM modulation. Finally, the bit loading algorithms use $P_{i}$ values that have not been equalized by the frequency domain equalizers, although this too would introduce error due to the channel estimation errors.

\section{B. Results and Discussion}

We first examine the effects of adding Gaussian noise to the subcarrier SNR values on the throughput performance of several adaptive bit loading algorithms. Results for the algorithms of Fox [2], the two algorithms of the authors [5, 6], and Leke \& Cioffi [3] were obtained. The first three algorithms all had similar results, therefore only the throughput results for one of the authors' algorithm [6] as well as for the algorithm of Leke $\&$ Cioffi [3] are presented in Fig. 3, while Figs. 4 and 5 show the average BER $\bar{P}$ and outage probability results for our algorithm [6]. The results are obtained when the variance of the Gaussian noise is either $\sigma^{2}=10,10^{2}, 10^{3}$, or $10^{4}$. Compared to the case where no Gaussian noise is added to the subcarrier SNR values, the throughput of the system decreases as the variance increases. In particular, the throughput curves shift to the right as the noise variance $\sigma^{2}$ increases. Moreover, except at low SNR values for $\sigma^{2}=10^{4}$, the algorithm of Leke \& Cioffi [3] performs relatively poorly.

Since most of the adaptive bit loading algorithms are close to the maximum achievable throughput given the maximum error constraint $[5,6]$, the addition of Gaussian noise to the subcarrier SNR values can either cause the system to violate the constraint (when $\hat{\gamma}^{(i)}>\gamma^{(i)}$ ) or decrease in throughput (when $\left.\hat{\gamma}^{(i)} \leq \gamma^{(i)}\right)$. Working under the assumption that $\bar{P} \geq P_{T}$ is not acceptable, when the former occurs, we set the throughput of the system to zero and record the number of times these violations occur. The fraction of realizations that are violations is shown in Fig. 5. When the latter occurs, the throughput and $\bar{P}$ are lower, as in Figs. 3 and 4 . Note that at low SNR values, $\sigma^{2}$ is large enough that the algorithm experiences violations every time. As the SNR increases, the violations respectively decrease. Other than the case of $\sigma^{2}=10$ violations occurred $100 \%$ of the time due to the SNR values being the same order of magnitude as the Gaussian noise.

The throughput, average BER $\bar{P}$, and outage probability re- sults of the authors' algorithm [6] using quantized subcarrier SNR values are presented in Figs. 6, 7, and 8, respectively. From Fig. 6 it can be observed that there is some degradation. However, the algorithm of Leke \& Cioffi [3] still has significantly lower throughout. For instance, at an SNR of $28 \mathrm{~dB}$, the difference in throughput for the authors' algorithm [6] between the ideal case and the case where the subcarrier SNR values are quantized to $2^{4}$ output levels is 25 bits/symbol. However, as the number of output levels increases, the throughput approaches that of the ideal case. For example, the difference in throughput for the authors' algorithm [6] between the ideal and a quantized subcarrier SNR employing $2^{10}$ output levels at an SNR of $28 \mathrm{~dB}$ is $1 \mathrm{bit} / \mathrm{symbol}$. Equivalently, the $\bar{P}$ curves in Fig. 7 also approach the ideal case when the number of output levels increases. This is due to the size of the granular error and relationship between $\hat{\gamma}^{(i)}$ and $\gamma^{(i)}$. If $\hat{\gamma}^{(i)}>\gamma^{(i)}$, the system will violate the maximum error constraint since the adaptive bit loading algorithms are close to the maximum achievable throughput, as seen in Fig. 8. On the other hand, if $\hat{\gamma}^{(i)} \leq \gamma^{(i)}$, the system will achieve a throughput and $\bar{P}$ less than the ideal case. The difference in performance is dependent on granular error. More output levels results in a smaller granular error and correspondingly a result closer to the ideal case.

\section{CONCLUSION}

Adaptive bit loading is an important technique to increase throughput while maintaining a satisfactory quality of transmission. Our results show a significant decrease in throughput when the adaptive bit loading algorithms use imperfect subcarrier SNR information. Therefore, the effects of imperfect SNR information cannot be ignored. However, this decrease varies depending on which adaptive bit loading algorithm is employed by the system. With respect to the four algorithms studied, the results showed that authors' algorithm [6] was the most robust when imperfect subcarrier SNR information was used, thus yielding the best throughput results.

\section{REFERENCES}

[1] IEEE Std. 802.11a, "Wireless LAN medium access control (MAC) and physical layer (PHY) specifications: High-speed physical layer in the 5 GHz band," Nov. 1999.

[2] B. Fox, "Discrete optimization via marginal analysis," Management Science, vol. 13, pp. 210-216, Nov. 1966.

[3] A. Leke and J. M. Cioffi, "A maximum rate loading algorithm for discrete multitone modulation systems," Proc. IEEE Global Telecommun. Conf., vol. 3, pp. 1514-1518, 1997.

[4] R. F. H. Fischer and J. B. Huber, "A new loading algorithm for discrete multitone transmission," Proc. IEEE Global Telecommun. Conf., vol. 1, pp. 724-728, 1996.

[5] A. M. Wyglinski, P. Kabal, and F. Labeau, "Adaptive bit and power allocation for indoor wireless multicarrier systems," Proc. Int. Conf. Wireless Commun., pp. 500-508, July 2003.

[6] A. M. Wyglinski, F. Labeau, and P. Kabal, "An efficient bit allocation algorithm for multicarrier modulation," Accepted to IEEE Wireless Commun. Network Conf., 2004.

[7] A. Leke and J. M. Cioffi, "Impact of imperfect channel knowledge on the performance of multicarrier systems," Proc. IEEE Global Telecommun. Conf., vol. 2, pp. 951-955, 1998.

[8] S. Ye, R. S. Blum, and L. J. Cimini, "Adaptive modulation for variablerate OFDM systems with imperfect channel information," Proc. 55th IEEE Veh. Technol. Conf. - Spring, vol. 2, pp. 767-771, 2002. 
[9] M. R. Souryal and R. L. Pickholtz, "Adaptive modulation with imperfect channel information in OFDM," Proc. IEEE Int. Conf. Commun., vol. 6, pp. 1861-1865, 2001.

[10] A. Czylwik, "Degradation of multicarrier and single carrier transmission with frequency domain equalization due to pilot-aided channel estimation and frequency synchronization," Proc. IEEE Global Telecommun. Conf., vol. 1, pp. 27-31, 1997.

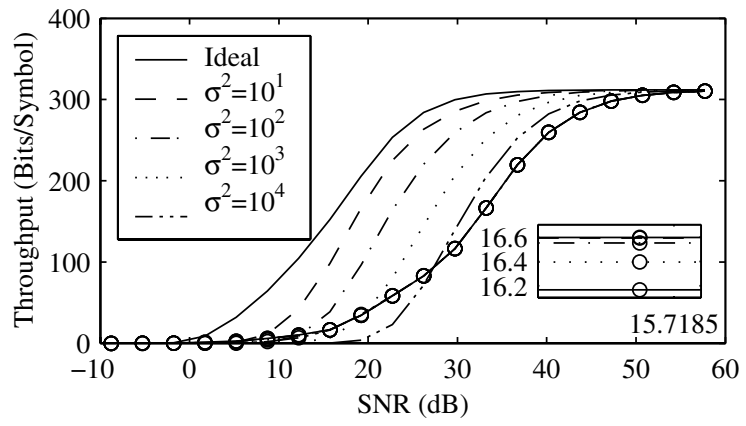

Fig. 3 Throughput of a multicarrier system employing adaptive bit loading algorithms of the authors [6] (no circles) and Leke \& Cioffi [3] (with circles) when Gaussian noise of variance $\sigma^{2}$ is added to the subcarrier SNR values.

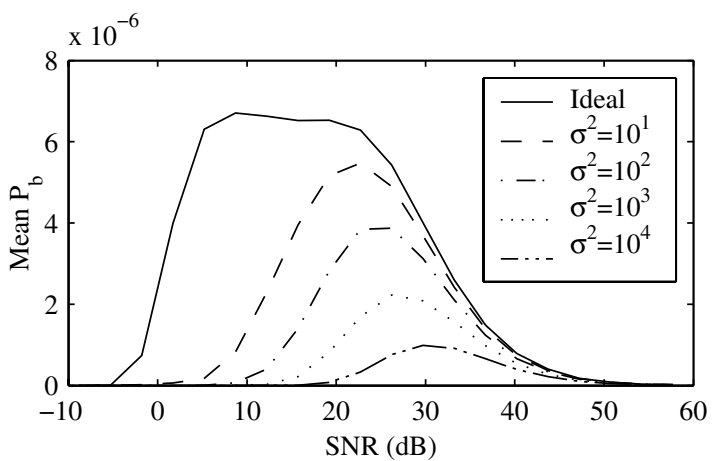

Fig. 4 Average BER of a multicarrier system employing the authors' adaptive bit loading algorithm [6] when Gaussian noise of variance $\sigma^{2}$ is added to the subcarrier SNR values.

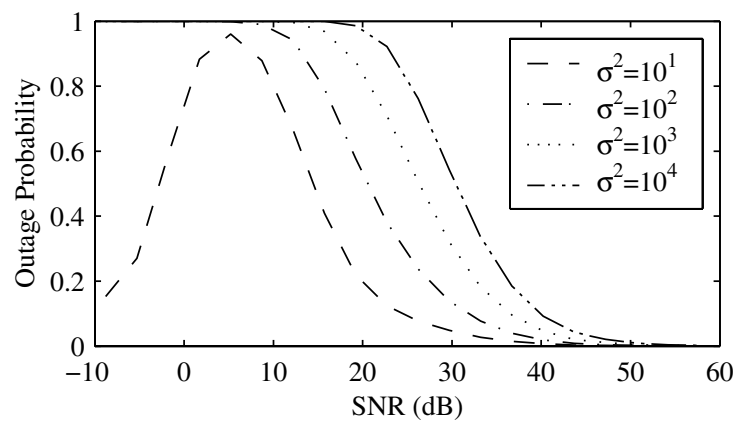

Fig. 5 Outage probability (fraction of realizations for which $\bar{P}>P_{T}$ ) of a multicarrier system employing the authors' adaptive bit loading algorithm [6] when Gaussian noise of variance $\sigma^{2}$ is added to the subcarrier SNR values.
[11] A. A. M. Saleh and R. A. Valenzuela, "A statistical model for indoor multipath propagation," IEEE J. Select. Areas Commun., vol. 5, pp. 128-137, Feb. 1987.

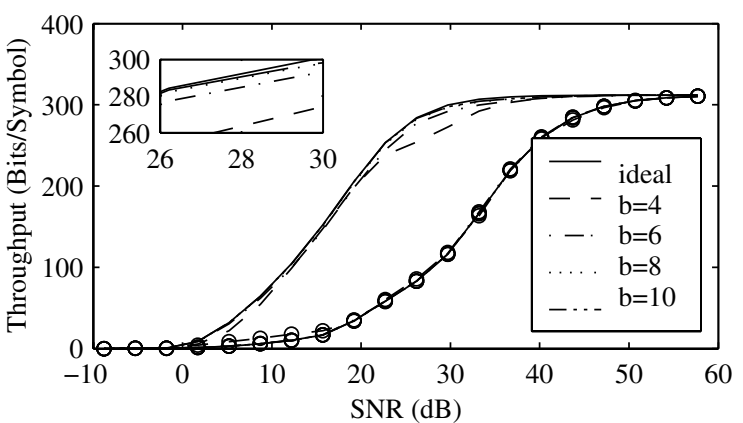

Fig. 6 Throughput of a multicarrier system employing adaptive bit loading algorithms of the authors [6] (no circles) and Leke \& Cioffi [3] (with circles) when the subcarrier SNR values are quantized with $2^{b}$ levels. Note the latter uses adapted quantization reproduction levels.

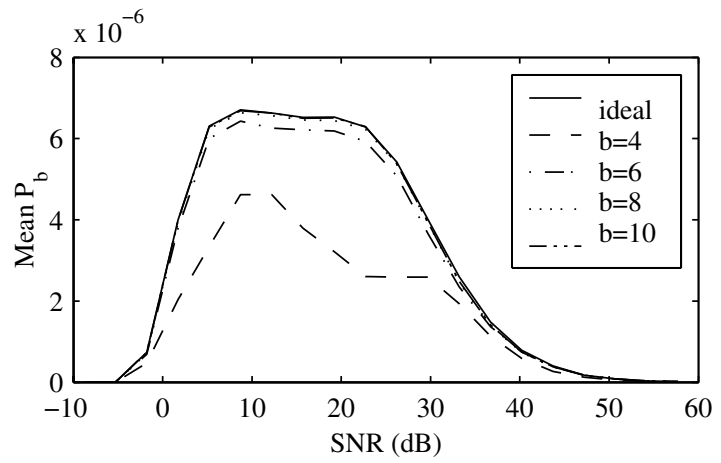

Fig. 7 Average BER of a multicarrier system employing the authors' adaptive bit loading algorithm [6] when the subcarrier SNR values are quantized with $2^{b}$ levels.

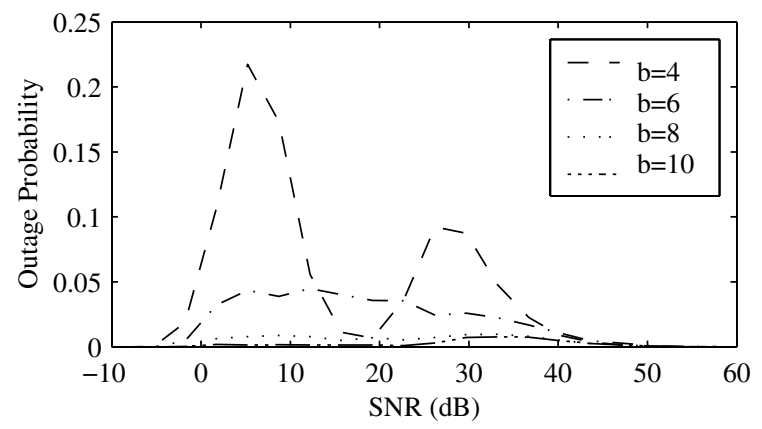

Fig. 8 Outage probability (fraction of realizations for which $\bar{P}>P_{T}$ ) of a multicarrier system employing the authors' adaptive bit loading algorithm [6] when the subcarrier SNR values are quantized with $2^{b}$ levels. 